\title{
The Distribution of Neutral Atomic Hydrogen in the Interacting Ringed Barred Spiral NGC $\mathbf{5 8 5 0}$
}

\author{
James L. Higdon \\ NRAO, P. O. Box O, Socorro, NM, 87801 \\ R. Buta \\ University of Alabama, Dept. of Astronomy, Box 870324, Tuscaloosa, \\ AL 35487-0324
}

\section{Introduction}

NGC 5850 is a large, nearby $\mathrm{SB}(\mathrm{r}) \mathrm{b}$ spiral that is an apparent member of the NGC 5846 group of galaxies (de Vaucouleurs 1975; Turner \& Gott 1976). The mean corrected redshift of this group is $\approx 1900 \mathrm{~km} \mathrm{~s}^{-1}$ (Haynes \& Giovanelli 1991). We have obtained broadband imaging and VLA HI interferometry of this galaxy as part of a general study of its properties. The most noteworthy characteristic of the galaxy is a severe distortion of the outer regions. This distortion is most likely due to an interaction with another member of the group, possibly NGC 5846 or its companion NGC $5846 \mathrm{~A}$, which are only $10^{\prime}$ to the northwest. In this paper we summarize some of the results of the optical and $\mathrm{HI}$ analysis, and highlight the effects of the interaction.

\section{CCD Imaging}

$B$ - and $I$-band images were obtained with the KPNO 0.9-m telescope in 1991 June with an ST1K CCD. Figure 1 shows the $B$ image (top left) and the $B-I$ color index map (top right). The galaxy is a classic barred spiral of the inner ring variety. There are at least three major spiral arms beyond the ring. The bar is fairly well-centered within the ring, but the outer arms are asymmetric. The color index map (where dark=blue, light=red) reveals that the inner ring is a zone of active star formation. In the bar, there is a miscentered nuclear pseudoring made of dust. This feature is linked to the dust lanes on the leading edges of the bar.

The central regions include a small secondary bar but little recent star formation. There is a weak nuclear ring or lens (Buta \& Crocker 1993). The nuclear dust pseudoring is larger than the secondary bar and has a different orientation. The secondary bar trails the axis of the primary bar by $67^{\circ}$, assuming the outer arms trail.

\section{Surface Photometry}

The orientation of the system was derived from ellipse fits to isophotes. In the outer regions, the axial ratio averages at 0.81 and the position angle at $166^{\circ}$. 

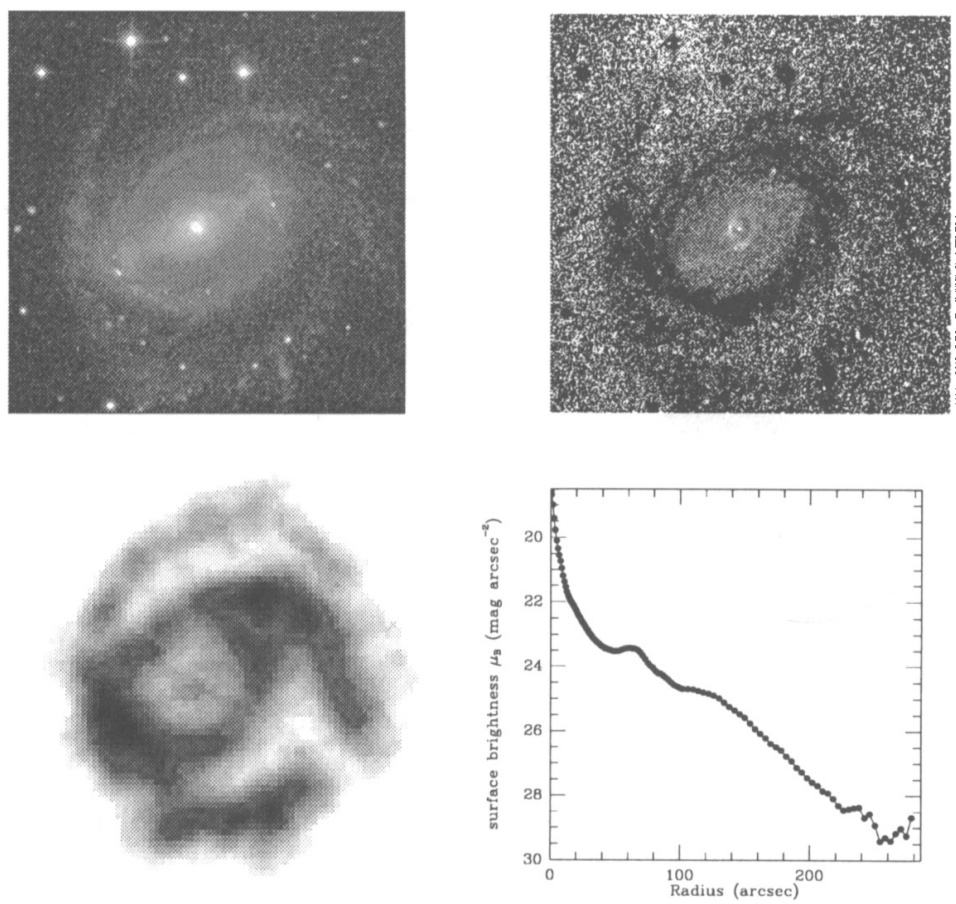

Figure 1. NGC 5850 in $B$-band (top left), $B-I$ (top right), and in 21-cm emission (bottom left). Profile at lower right is an ellipse average.

The faintest isophotes are miscentered nearly due west by $16^{\prime \prime}$. An azimuthallyaveraged surface brightness profile within an ellipse having this shape and centered on the nucleus is shown in Figure 1 (bottom right). In spite of the asymmetry, a clear exponential decline is in evidence in the outer regions. Fourier amplitudes and phases for the $1 \theta$ component of the light distribution indicate that the effects of the asymmetry are very large beyond $1.5 r_{25}$, where $r_{25}$ is the radius of the $\mu_{B}=25.0 \mathrm{mag} \operatorname{arcsec}^{-2}$ isophote. Within $0.75 r_{25}$, there is very little asymmetry.

\section{HI Observations}

The HI observations were obtained with the VLA in C and D configurations in 1987 and 1991. Figure 1 (bottom left) shows the combined C and D array HI distribution at a resolution of $28^{\prime \prime} \times 25^{\prime \prime}$, based on the natural weighting procedure in AIPS. The inner ring and spiral arms are clearly seen in the HI, and in fact the HI follows the optical structure very closely. There is low-density gas inside the inner ring with a slight peak in the nucleus. Figure 2 (left) shows that the HI velocity field is disturbed on the west side. The rotation curves for the two halves of the major axis are shown in Figure 2 (right). These were calculated for a fixed inclination of $37^{\circ}$, and are based on data within $45^{\circ}$ of the 

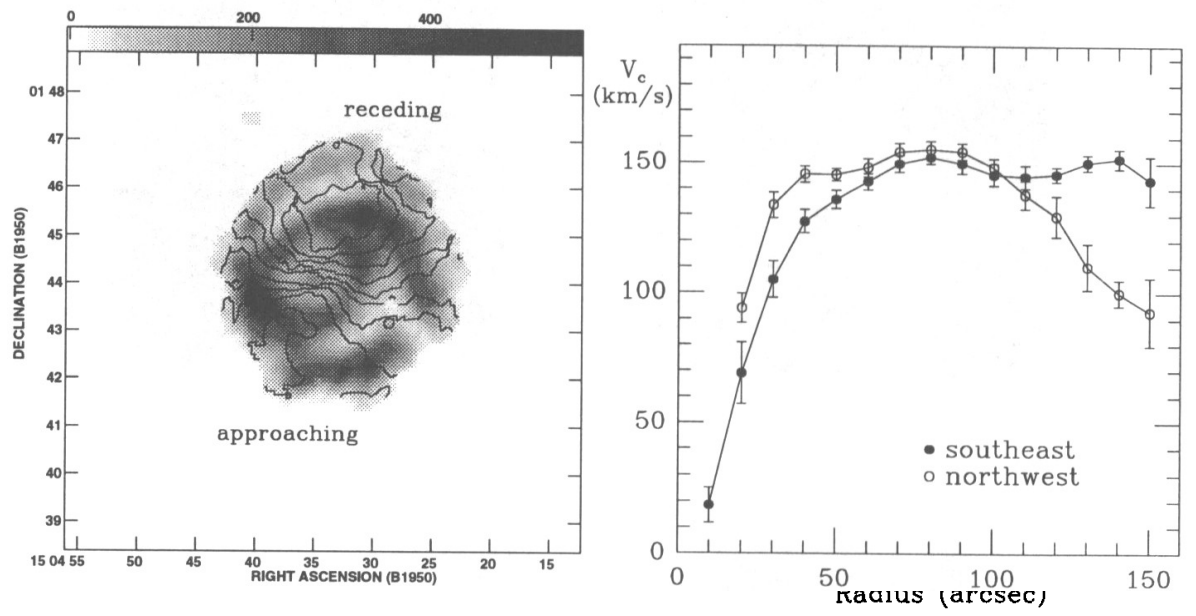

Figure 2. Velocity field and rotation curves of NGC 5850 . Velocity contours are separated by $20 \mathrm{kms}^{-1}$. Greyscale is in $\mathrm{Jy} \mathrm{m} \mathrm{s}^{-1}$ beam ${ }^{-1}$.

major axis. The disagreement between the two halves is probably due to the interaction. The falling section is on the northwest side where the asymmetry is strongest. The derived position angle, systemic velocity, and rotation center for the two sides combined were $338^{\circ} \pm 2^{\circ}, 2535 \pm 10 \mathrm{~km} \mathrm{~s}^{-1}$, and $12^{\prime \prime} 5$ West, $7 ! 5$ south of the pointing center $(150435.50,+014417.0)(1950)$, respectively.

\section{Conclusions}

NGC 5850 is a striking example of an interacting barred spiral. The perturber may be NGC $5846 \mathrm{~A}$, a compact $\mathrm{E}$ galaxy of comparable redshift superposed within the envelope of NGC 5846. Further modeling of the kinematics should provide an idea of the history of the interaction.

\section{References}

Buta, R. \& Crocker, D. 1993, AJ, 105, 1344

de Vaucouleurs, G. 1975, in Stars and Stellar Systems, Vol. IX, A. Sandage, M.

Sandage, \& J. Kristian, Chicago: Univ. of Chicago Press, 557

Turner, E. \& Gott, R. 1976, ApJS, 32, 409

Haynes, M. \& Giovanelli, R. 1991, AJ, 102, 841 